\title{
Consumo de anime en la población universitaria de Cartagena
}

\author{
Francisco Javier Arias Álvarez ${ }^{*}$ Natalia Robledo Rodríguez ${ }^{* *}$, Tania Lucía Cobos ${ }^{* * *}$
}

DOI: https://doi.org/10.33571/revistaluciernaga.v12n23a3

\section{Resumen}

Esta investigación presenta un análisis sobre el consumo de anime en la población universitaria de Cartagena (Colombia). Se encontró que la mayoría de los participantes conciben este tipo de animación japonesa como una guía para su propia vida; se identifican con las historias, protagonistas y los valores de la responsabilidad, perseverancia, espíritu luchador y amistad, que caracterizan a estas producciones.

Palabras claves: Cartagena de indias; anime; exposición; influencia; otaku.

Recibido. Febrero 21, 2020

Aceptado. Marzo 03,2020

\begin{abstract}
*Comunicador social de la Universidad Tecnológica de Bolívar. Integrante del semillero de investigación Medios Masivos y Productos Culturales.. Orcid: https://orcid.org/0000-0001-6817-0338, e-mail: fran.arias. alvarez@gmail.com

** Comunicadora social de la Universidad Tecnológica de Bolívar. Integrante del semillero de investigación Medios Masivos y Productos Culturales. Su aporte y el del primer autor fue la construcción teórica, apoyo en la construcción del instrumento de recolección de datos y trabajo de campo. Orcid: https://orcid.org/0000-0002-4379-2226; e-mail: nataliarobledo951109@gmail.com
\end{abstract}

***Doctora en Comunicación y Periodismo de la Universitat Autònoma de Barcelona (ES). Maestra en Ciencias en Comunicación del Tecnológico de Monterrey (MX); Especialista en Periodismo Electrónico de la Universidad Pontificia Bolivariana, comunicadora social y periodista de la Universidad del Norte. Profesora-investigadora del programa de Comunicación Social Universidad Tecnológica de Bolívar. Su función en este trabajo fue el diseño de la metodología, la construcción del instrumento, el trabajo de campo, y la dirección de la redacción del artículo. Orcid: https://orcid.org/0000-00018348-3631; e-mail: tcobos@utb.edu.co 


\title{
Consumption of anime in the university population in Cartagena
}

\author{
Francisco Javier Arias Álvarez*, Natalia Robledo Rodríguez ${ }^{* *}$, Tania Lucía Cobos ${ }^{\star * *}$
}

DOI: https://doi.org/10.33571/revistaluciernaga.v12n23a3

\begin{abstract}
This research presents an analysis on anime consumption in the university population of Cartagena (Colombia). It was found that most of the participants conceive this type of Japanese animation as a guide for their own life; they identify with the stories, protagonists and the values of responsibility, perseverance, a fighting spirit and friendship that characterize these productions.
\end{abstract}

Keywords: Cartagena; anime; exposure; influence; otaku.

Received. February 21, $2020 \quad$ Accepted. March 03,2020

\footnotetext{
* Comunicador social de la Universidad Tecnológica de Bolívar. Integrante del semillero de investigación Medios Masivos y Productos Culturales. Their contribution to the article was: the theoretical construction, they collaborated in the construction of the instrument, they supported the field work somewhat, they tabulated and analyzed the data in this paper and wrote the conclusions. Orcid: https://orcid.org/0000-0001-6817-0338, e-mail: fran.arias.alvarez@gmail.com

** Comunicadora social de la Universidad Tecnológica de Bolívar. Integrante del semillero de investigación Medios Masivos y Productos Culturales. Their contribution to the article was: the theoretical construction, they collaborated in the construction of the instrument, they supported the field work somewhat, they tabulated and analyzed the data in this paper and wrote the conclusions. Orcid: https://orcid.org/0000-0002-4379-2226; email: nataliarobledo951109@gmail.com

*** Doctor in Communication and Journalism from the Autonomous University of Barcelona (ES). Master of Science in Communication from Tecnológico de Monterrey (MX); Specialist in Electronic Journalism from the Universidad Pontificia Bolivariana, social communicator and journalist from the Universidad del Norte. Professor-researcher of the Social Communication program Universidad Tecnológica de Bolívar. Her role in this work was the design of the methodology, the construction of the instrument, the field work, and the direction of the writing of the article. Orcid: https://orcid.org/00000001-8348-3631; e-mail: tcobos@utb.edu.co
} 


\title{
Consumo de anime na população universitária de Cartagena
}

\author{
Francisco Javier Arias Álvarez ${ }^{*}$ Natalia Robledo Rodríguez ${ }^{* *}$, Tania Lucía Cobos ${ }^{* * *}$
}

DOI: https://doi.org/10.33571/revistaluciernaga.v12n23a3

\section{Resumo}

Esta pesquisa apresenta uma análise sobre o consumo de anime na população universitária de Cartagena (Colômbia). Verificou-se que a maioria dos participantes concebe este tipo de animação japonesa como um guia para sua própria vida; identificam-se com as histórias, protagonistas e valores de responsabilidade, perseverança, espírito de luta e amizade que caracterizam estas produções.

Palavras chave: Cartagena das índias; anime; exposição; influência; otaku.

\footnotetext{
* Comunicador social da Universidade Tecnológica de Bolívar. Membro do Núcleo de Pesquisa em Meios de Comunicação de Massa e Produtos Culturais Orcid: https://orcid.org/0000-0001-6817-0338, e-mail: fran.arias.alvarez@gmail.com

** Comunicador social da Universidade Tecnológica de Bolívar. Membro do Núcleo de Pesquisa em Mídia de Massa e Produtos Culturais. A contribuição dele e do primeiro autor foi a construção teórica, suporte na construção do instrumento de coleta de dados e trabalho de campo. Orcid: https://orcid.org/0000-0002-4379-2226; e-mail: nataliarobledo951109@gmail.com

*** Doutor em Comunicação e Jornalismo pela Universidade Autônoma de Barcelona (ES). Mestre em Ciências da Comunicação pela Tecnológico de Monterrey (MX); Especialista em Jornalismo Eletrônico pela Universidad Pontificia Bolivariana, comunicadora social e jornalista pela Universidad del Norte. Professor-pesquisador do programa de Comunicação Social Universidad Tecnológica de Bolívar. Sua função neste trabalho foi o desenho da metodologia, a construção do instrumento, o trabalho de campo e a direção da redação do artigo. Orcid: https://orcid.org/0000-0001-8348-3631; e-mail: tcobos@utb.edu.co
} 


\section{Introducción}

El anime es uno de los productos audiovisuales de Japón, país del sol naciente, que goza de una gran popularidad y consumo en el mundo. Tanto la estética que propone como la variedad de historias y universos que ofrece, permite la proliferación de esta industria acaparando tanta atención como ingresos posibles. En 2018, la industria del anime representó el 10\% del PIB de la economía nipona, entre la venta y el consumo de manga y anime, así como sus derivados en marketing (Ortega en Agencia de Noticias Univalle, 2018). Asimismo, en un informe de la Asociación de Animaciones Japonesas - AJA, en el 2018 el mercado global del anime fue valorado en 21.814 billones de yenes (alrededor de 19,9 mil millones de dólares) (IGN Latinoamérica, 2019).

Temporada tras temporada salen en emisión decenas de anime los cuales, ya sea por lo novedoso de sus temáticas o la singularidad en la forma de abordarlas, movilizan grandes séquitos de fans quienes demuestran su afición a través del consumo de todo tipo de merchandising y generando comunidad.

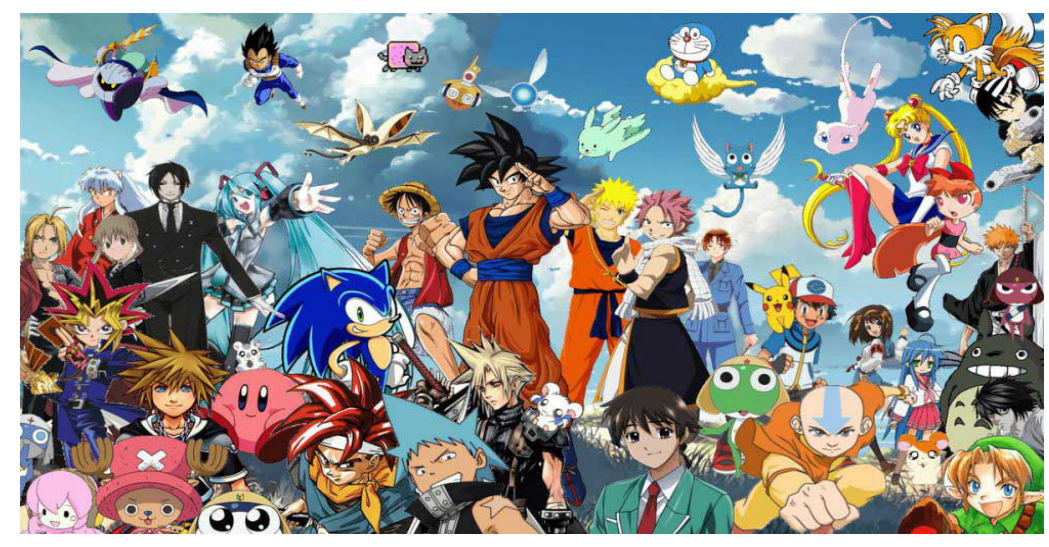

Tomado de : https://www.viaje.jp/cultura/anime-japon/anime iconicos japon.html

En Latinoamérica el tema ha sido abordado previamente en estudios como Análisis de la influencia cultural del anime japonés en la recepción comunicológica de estudiantes de primer y tercer semestre de Diseño Gráfico de la Universidad de Guayaquil de Intriago Córdova (2018), orientado a determinar cómo el anime influía en la recepción comunicológica de estudiantes a través de la construcción de sus imaginarios sociales. Influencia de los animes japoneses en los jóvenes de la asociación cultural A-Shinden Puno de Galindo Velásquez (2017), el cual encontró una influencia significativa del anime en los jóvenes de dicha asociación cultural con respecto al conocimiento, imitación y prácticas tradicionales de la cultura japonesa. Influencia cultural del anime y manga japonés en México de Romero Quiroz (2012), que evidenció que los consumidores de este tipo de contenidos generan nuevos referentes de identidad cultural, tanto individual como grupal, sin dejar de lado la propia identidad y/o contexto de los mexicanos. Y Japón hecho en Venezuela. Influencia cultural japonesa en la cosmovisión del mundo de los jóvenes en Venezuela a través de la televisión y el anime de Conejo Quinche (2011), el cual señaló la posibilidad de comprobar que las barreras interpretativas entre personas con diferentes idiomas y culturas pueden desaparecer, a la vez que se generan diversos procesos en los que desarrollan emociones y se adaptan a los nuevos modos de comunicación, tomando como referencia programas de televisión de origen japonés como el anime. 
Se encontraron otros estudios como El anime y la apropiación de la cultura japonesa por parte del otaku barranquillero de Pichón Ayazo (2012),en éste se analizó cómo los fanáticos barranquilleros del anime y el manga se apropian de la cultura e idioma de los productos que consumen y viven su identidad otaku a partir de sus prácticas cosplay [1], realización de eventos y adaptación de términos japoneses a su idioma cotidiano. También, Apropiación de dramas coreanos en un grupo de jóvenes de Cartagena de Indias de Zarco Quintero (2017).

\section{El anime}

En cuanto a la definición de anime nos aproximamos a través de lo conceptualizado por Cobos (2010, p. 7), quien afirma que es "el término occidental con el que se conoce a la producción de dibujos animados hechos en Japón". Su formato de distribución puede ir desde animación para la televisión, películas para cine y material directo a DVD o Bluray (llamadas OVAs -Original Animation Video-). Si bien originalmente el término anime es una abreviación del término "animēshon", denominación con la que la cultura nipona se refiere a los dibujos animados en general, independientemente de su estilo y nacionalidad, cruzando el océano y desde occidente, la palabra anime hace referencia exclusivamente a la animación realizada en Japón (Arciniegas y Mora, 2010, p. 15).

Al hablar de anime cabe citar la existencia de otro vocablo: el manga. Según Arciniegas y Mora (2010, p. 15) "se trata de dos términos cercanos, pero disímiles, puesto que el manga hace referencia a los cómics japoneses mientras que el anime a las animaciones, muchas veces de dichos cómics". Esto puede ser entendido debido a que el anime, al ser un producto cultural con una gran industria audiovisual detrás, sus obras pueden ser desde ideas originales hasta adaptaciones de todo tipo. Así, un anime puede tener su origen en el manga, novelas ligeras, videojuegos, juegos de mesa, películas, literatura universal o ser una concepción original de su creador.

A su vez, existe otro término bastante cercano al anime y esta vez hace referencia a la forma en que se denominan su grupo de aficionados: otaku. Cobos (2010, p. 24) afirma que "la llegada del anime y el manga a América Latina ha generado también, como en el resto de occidente, el nacimiento de una nueva subcultura que se autodenomina otaku". Éste "es el término japonés empleado para designar a un fanático obsesivo del anime y manga" (Pichón, 2012, p. 30). Según Arciniegas y Mora (2010, p. 29) es un término japonés usado para referirse de forma peyorativa a las personas introvertidas con aficiones malsanas, no propiamente del anime/manga. En occidente, el vocablo hace referencia sólo al aficionado del anime/manga y tienen una connotación más bien ambigua debido a que muchos fanáticos consideran un orgullo ser denominados de esa forma, y otros, al saber la acepción original, eluden etiquetarse de esta forma (Pichón 2012, p. 32).
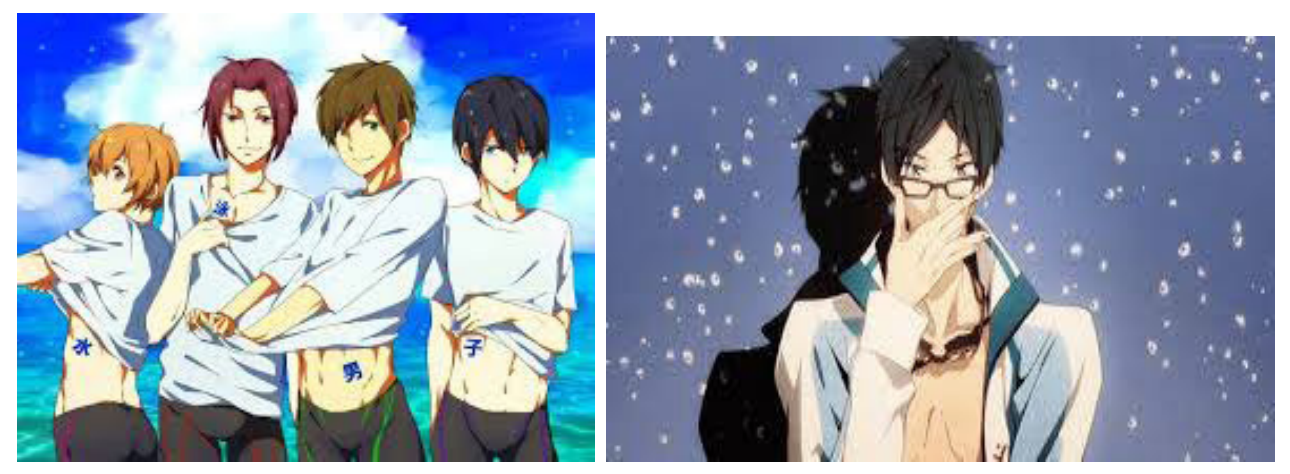

Tomado de: https://www.facebook.com/pg/swimminganimeFree/posts/ 
Retomando, una de las grandes características del anime es su vasto abanico de temáticas, cuestión que desemboca en un amplio y extenso número de géneros. De lo expuesto por Álvarez de Moll (2008 en Arciniegas y Mora, 2010, p. 16) se infiere que dichos géneros se dividen en dos grandes grupos. Por un lado, la demografía de su audiencia, y por el otro, el tipo de historia.

Según la demografía estos pueden ser: kodomo, dirigido a los niños; shōnen, para chicos adolescentes; shōjo, para chicas adolescentes; seinen, dirigido a adultos hombres; y josei dirigido a mujeres adultas. Esta distinción de géneros por edades básicamente es heredada de la cultura del manga, pues estos se distribuyen en revistas con distintas obras por edición atendiendo al tipo de público. Así, por ejemplo, Shūkan Shōnen Jump atiende sólo al público shōnen, es decir, adolescentes masculinos y la Bessatsu Friend dirigida al público shōjo, dicho de otra forma, adolescentes femeninas.

En cuanto al tipo de historia, los géneros pueden ser: nekketsu; de altas dosis de acción, peleas y aventuras; slice of life sobre recuentos de la vida; mecha, robots gigantes que luchan contra los kaiju (monstruos también gigantes); spokon, de carácter deportivo; harem/harem inverso, chico rodeado de chicas o viceversa; magical girlfriend, sobre personajes comunes relacionándose con seres fuera de lo común; mahō shōjo, de chicas con poderes mágicos; ecchi, sobre situaciones con tintes picantes o eróticas; hentai explícitamente pornográfico; hibi, personajes pequeños y cabezones; kemono, personajes con rasgos y características animales; isekai, sobre humanos normales atrapados en mundos paralelos; shōjo ai/yuri y shōnen ai/yaoi, historias de romance homosexual de mujeres y hombres respectivamente.

Cabe mencionar que el anime también toca géneros comunes o presentes en las obras de carácter universal; tales como el ciberpunk, sobre distopías futuristas; meitantei o género detectivesco, romakome o romance, steampunk sobre mundos tecnológicos de la revolución industrial, el sci-fi sobre mundos futuristas o tecnológicos y gore o ficciones de carácter sangriento y violento.

\subsection{El anime en Colombia}

En Colombia la televisión ha cumplido un papel importante en la difusión del anime. Pichón (2012, p. 29), registra las primeras emisiones de animación japonesa en televisión nacional en la década de los 80s con series como Astroboy (1980) y Fuerza G (1982) por el canal Cadena 1 y Meteoro (1984), Heidi (1985), La Abejita Maya (1985) y Candy Candy (1988) por la Cadena 2. En 1987 inició la emisión de Cuentos de los Hermanos Grimm, anime con más de 20 años en la parrilla de programación siendo, por tanto, el más longevo en la TV nacional (Shock, 2019).

A partir de la década de los 90 s la televisión colombiana experimentó varios cambios. Por un lado, la evolución de la televisión pública que se refleja en la existencia de los canales públicos nacionales Señal Colombia, Canal Institucional y Canal 1 (público-privado), también la aparición de la televisión privada y con ésta los canales Caracol Televisión y RCN Televisión. Asimismo, la diversificación de los canales regionales tanto públicos como privados, siendo algunos de estos CityTV, Telecaribe, Telepacífico, entre otros (Banrepcultural, s.f). De la misma manera, la llegada de la televisión por suscripción que permitió sintonizar canales extranjeros como XHGC Canal 5 (México), América TV (Perú), Locomotion (Argentina), entre otros. 
Todo lo anterior facilitó el incremento de series de anime en la pantalla televisiva y se emitieron varias que alcanzaron gran popularidad entre las audiencias: Supercampeones (1993), Los Caballeros del Zodíaco (1993), Dragon Ball (1996), Sailor Moon (1996), Dragon Ball Z (1998), Pokémon (1999), Ranma 1/2 (1999), entre otras. Pero esta popularidad también implicó polémicas, ya que los contenidos de algunas chocaban con el contexto cultural nacional en varios sentidos, por ejemplo, la abierta relación lésbica entre las sailors scouts Haruka y Michiru en Sailor Moon; la transformación de Ranma Saotome en hombre si se mojaba con agua caliente o en mujer si se mojaba con agua fría, en Ranma 1/2; la violencia, sangre o el uso de palabras como destazar en Samurai X (Ruroni Kenshin), entre otros (El Tiempo, 2001).

En esta década que va corriendo, la animación japonesa también es consumida en el país desde servicios de streaming, tanto pagos como Crunchyroll o Netflix, o de consumo alegal como AnimeFLV, JkAnime, AnimeID, etc. Estas plataformas ofrecen ventajas diferenciales con respecto a la televisión tradicional como el consumo asincrónico en tandas, el visualizar subtitulados los nuevos capítulos en el mismo momento o pocas horas después de su estreno en Japón, o ver series que nunca se han licenciado para el país o que dejaron de emitirse.

Aún así, la televisión en general sigue cumpliendo un papel relevante en la difusión del anime en el país, quizá no tan a la vanguardia como las plataformas previamente mencionadas, pero sí con propuestas interesantes. Ejemplo de esto es Señal Colombia que en su sección "En Cine nos Vemos" ha emitido filmes de Studio Ghibli. Obras como El viento se levanta, Mis vecinos los Yamada, Ponyo en el acantilado, Lupin III en el Castillo de Cagliostro, Recuerdos del ayer, entre otras, han tenido su respectiva función en este canal, también Candy Candy, considerada un clásico. Por su parte, Canal 1 en su franja matinal de los fines de semana ha emitido series como Dragon Ball Super, Supercampeones, Los Caballeros del Zodíaco y Sailor Moon, así como Canal Claro ha tenido en su parrilla también a Los Caballeros del Zodíaco y tres de las series de Anime Marvel (X-Men, Iron Man y Wolverine).

\subsection{El enfoque de la Teoría del Cultivo o los Indicadores Culturales}

La investigación se abordó desde el enfoque de la Teoría del Cultivo o Indicadores Culturales, la cual busca determinar los efectos y/o consecuencias en los receptores expuestos a la programación televisiva creada para entretenerlos. Según Igartua y Humanes (2004, p. 267-270), esta teoría se centra en analizar la manera cómo los espectadores, a partir de lo que visualizan en la pantalla, pueden llegar a estructurar y generar un refuerzo en sus creencias, orientaciones sociales y perspectivas de la vida real mediante un sistema que envía mensajes estables y constantes, afianzando una forma de ver y entender su entorno social. De aquí que "la noción de cultivo se refiera a la contribución independiente que aporta la televisión a las concepciones que las personas se forman del mundo social como producto de una exposición sistemática, acumulativa y de largo plazo a sus contenidos, que son repetitivos y convencionales" (D’Adamo et al., 2007, p. 100).

Asimismo, D'Adamo et al. (2007, p. 101-103), indica que el interés de los pioneros de esta teoría radica especialmente en las consecuencias de las percepciones del entorno social, actitudes y opiniones de la población frente al uso, consumo y grado de exposición a la televisión. Para probar su hipótesis y supuestos básicos, se dedicaron a tres líneas de investigación denominadas Análisis Institucional, Análisis del Sistema de Mensajes y el Análisis del Cultivo. En estas tres etapas, los investigadores exploraron la producción y distribución de los mensajes de la televisión, examinaron qué modelo de la realidad social difundió la televisión e indagaron si los contenidos de esta influyen sobre la percepción pública. Para esta última, consideraron como variable fundamental el grado de aculturación. 
Gerbner y su equipo de trabajo realizaron un análisis de contenidos en el que lograron señalar que "hay ciertas imágenes y valores que se repiten constantemente en los diversos géneros y programas", (Gerbner y Gross, 1983 en Lozano, 2007, p. 125). La investigación señaló que, aunque no hay evidencia del impacto de los contenidos de los medios en la audiencia, sí se puede considerar válida la influencia de estos en ella, teniendo en cuenta tres imágenes y valores (supuestos básicos) recurrentes en los contenidos televisivos: la violencia social, los estereotipos sobre grupos demográficos y la homogenización de la ideología política (Lozano, 2007, p. 125-126).

Según Lozano (2007), respecto a la violencia televisiva (el primer supuesto), la probabilidad que el televidente asuma una actitud agresiva al finalizar un programa violento es baja, pero sostiene que para que este se vea influenciado por la exposición a dicho contenido y que este actúe como un detonador de conductas violentas, el individuo tendría que estar condicionado por su entorno (pobreza, desempleo, carencia de educación, familia desintegrada, etcétera). "Sin embargo, lo anterior no significa que las constantes imágenes de asesinatos, pleitos, destrucción, accidentes, balaceras y agresiones verbales que desbordan la televisión comercial sea inofensiva para los televidentes" (p. 127-128).

Sobre los estereotipos de grupos demográficos, Gerbner y sus colaboradores concluyeron que, "si bien no se puede afirmar que hay una relación causal entre el contenido de los anuncios y los problemas sociales como violaciones, trastornos alimenticios y discriminación en los centros de trabajo, tampoco contribuyen a disminuir las concepciones erróneas" (en Lozano, 2007, p. 131132). Por último, el tercer supuesto apunta a la homogenización de la ideología política, "siendo uno de los efectos más importantes del sistema de envío de imágenes y mensajes que llegan de igual manera (por la televisión) a los diferentes hogares creando una homogenización de valores, creencias y visiones del mundo, en grupos sociales que, por sus características demográficas, sociales, económicas y raciales deberían demostrar diferencias significativas", según Lozano (2007, p. 133). De esto último, los pioneros de la teoría del Cultivo señalaron que, entre mayor exposición de la audiencia a este tipo de contenidos, mayor será su predisposición para desarrollar visiones de la realidad, de la vida y la sociedad, similares a las representaciones en la televisión.

La investigación sobre la teoría de los Indicadores Culturales o del Cultivo "halló que la televisión influye sobre las actitudes, creencias y orientaciones políticas de la población", esto debido a que la televisión tiende a reproducir contenidos con valores homogenizados de manera que la audiencia conciba el mundo social de una manera semejante, con sesgo y estereotipos, de acuerdo con D'Adamo et al. (2007, p. 109).

El eje principal de este documento, al ser un producto derivado de una investigación sobre la relación entre el anime y su consumo por parte de la población universitaria de Cartagena de Indias (Colombia), apunta al análisis de los hallazgos desde el enfoque de la teoría del Cultivo, con la finalidad de entender cómo la exposición a la animación japonesa configura la cosmovisión de la audiencia universitaria cartagenera. Para ello, es necesario distinguir el tipo de contenidos anime más consumidos por estos y su relación con su concepción del mundo, así como identificar la influencia que ha tenido dicha exposición a estos contenidos japoneses en el estilo de vida de los mismos.

\section{Metodología}

Este estudio deriva del proyecto de investigación matriz "Usos y gratificaciones del consumo de animación japonesa en la población universitaria de Cartagena de Indias" desarrollado por el semillero Medios Masivos y Productos Culturales del programa de Comunicación Social de la 
Universidad Tecnológica de Bolívar, por lo que hereda la metodología implementada por éste y toma una porción de los hallazgos y genera sus particulares categorías de análisis encontrando su propia personalidad y distinción a la hora de interpretar los resultados.

La investigación matriz se planteó con un enfoque mixto y de alcance exploratorio y descriptivo, mismo que conserva este estudio. Hernández, Fernández y Baptista (2014, p. 534) afirman que este enfoque implica "un conjunto de procesos sistemáticos, empíricos y críticos de investigación e implican la recolección y el análisis de datos cuantitativos y cualitativos, así como su integración y discusión conjunta, para realizar inferencias producto de toda la información recabada (metainferencias) y lograr un mayor entendimiento del fenómeno bajo estudio". A su vez, su alcance exploratorio obedece a que se abordó o examinó un tema poco estudiado (Hernández, Fernández y Baptista, 2014, p. 91). Finalmente, su alcance descriptivo ya que implicó "describir fenómenos, situaciones, contextos y sucesos; esto es, detallar cómo son y se manifiestan [...] se busca especificar las propiedades, las características y los perfiles de personas, grupos, comunidades, procesos, objetos o cualquier otro fenómeno que se someta a un análisis" (Hernández, Fernández y Baptista, 2014, p. 92).

El instrumento que se aplicó fue una encuesta mixta compuesta 31 preguntas, enla que se recopilaron datos demográficos, formas y frecuencias de consumo, posicionamiento, usos, gratificaciones e influencia. Para ello, algunas preguntas tenían opción de respuesta, y en otras, contestación abierta. La encuesta estuvo disponible en línea en SurveyMonkey de marzo a mayo de 2019. La muestra, por tanto, se construyó a conveniencia a partir de la accesibilidad y proximidad de los sujetos para los investigadores. Finalmente, para incentivar la participación en su diligenciamiento, se sortearon dos membresías premium de Crunchyroll.

Para este análisis se estipularon las siguientes categorías: 1. Exposición al anime (edad en la que empezaron a ver anime y razones por las que empezaron a verlo), 2. Posicionamiento de anime (anime favoritos y géneros de anime), 3. Frecuencia de consumo (momento del día en el que ven anime y tiempo dedicado en horas y días a ver anime) y 4. Percepción de impacto del consumo (ver anime te ha motivado a, e impacto que el anime ha tenido en tu vida).

\section{Hallazgos}

La encuesta aplicada obtuvo por todo 350 respuestas válidas, es decir, que diligenciaron el cuestionario en su totalidad. En la Tabla 1 se detalla la conformación de la muestra:

\section{Tabla 1. Participantes}

\begin{tabular}{|c|c|c|c|c|c|}
\hline Rango de edad & Número & Porcentaje & Estado civil & Número & Porcentaje \\
\hline De 17 a 19 años & 120 & $34.3 \%$ & Soltero/a & 327 & $93.4 \%$ \\
\hline De 20 a 25 años & 177 & $50.6 \%$ & Unión libre & 16 & $4.6 \%$ \\
\hline De 26 a 30 años & 30 & $8.6 \%$ & Casado/a & 6 & $1.7 \%$ \\
\hline De 31 a 35 años & 15 & $4.3 \%$ & Divorciado/a & 1 & $0.3 \%$ \\
\hline De 36 a 40 años & 4 & $1.1 \%$ & & & \\
\hline De 41 a 45 años & 4 & $1.1 \%$ & & & \\
\hline Total & 350 & $100 \%$ & Total & 350 & $100 \%$ \\
\hline
\end{tabular}




\begin{tabular}{lcc}
\hline \multicolumn{1}{c}{ Sexo } & Número & Porcentaje \\
\hline Femenino & 139 & $39.7 \%$ \\
Masculino & 208 & $59.4 \%$ \\
No declara & 3 & $0.9 \%$ \\
\hline Total & 350 & $100 \%$ \\
\hline
\end{tabular}

\begin{tabular}{ccc}
\hline Rol & Número & Porcentaje \\
\hline Docente & 23 & $6.6 \%$ \\
Estudiante & 327 & $93.4 \%$ \\
& & \\
\hline Total & 350 & $100 \%$ \\
\hline
\end{tabular}

Fuente: elaboración propia

La Tabla 1 expone las características demográficas de los participantes. Inicialmente, uno de los datos a destacar es que más de la mitad de la muestra es masculina (59.4\%), así mismo que la edad mayoritaria va de 20 a 25 años (50.6\%). Sobre el rol desempeñado en la universidad el $93.4 \%$ de los encuestados son estudiantes y soletros. Puede decirse entonces que el encuestado promedio de la muestra poblacional es un estudiante masculino, soltero y con una edad oscilante entre 20 y 25 años.

Tabla 2. Exposición al anime

\begin{tabular}{|c|c|c|c|}
\hline \multicolumn{4}{|c|}{ Edad en la que empezaron a ver anime } \\
\hline Respuesta & Resultado & \multicolumn{2}{|l|}{ Porcentaje } \\
\hline De 6 a 9 años & 157 & \multicolumn{2}{|l|}{$44.9 \%$} \\
\hline De 10 a 13 años & 102 & \multicolumn{2}{|l|}{$29.1 \%$} \\
\hline De 14 a 17 años & 70 & \multicolumn{2}{|l|}{$20 \%$} \\
\hline De 18 a 21 años & 17 & \multicolumn{2}{|l|}{$4.9 \%$} \\
\hline De 22 años en adelante & 4 & \multicolumn{2}{|l|}{$1.1 \%$} \\
\hline Total & 350 & \multicolumn{2}{|l|}{$100 \%$} \\
\hline \multicolumn{4}{|c|}{ Razones por las que empezaron a ver anime } \\
\hline \multicolumn{2}{|c|}{ Respuesta } & Resultado & Porcentaje \\
\hline \multicolumn{2}{|c|}{ Porque las tramas eran diferentes a otro tipo de caricaturas } & 206 & $58.8 \%$ \\
\hline \multicolumn{2}{|c|}{ Por el estilo y estética del dibujo } & 168 & $48 \%$ \\
\hline \multicolumn{2}{|c|}{ Porque eran más entretenidos que otro tipo de caricaturas } & 164 & $46.8 \%$ \\
\hline \multicolumn{2}{|c|}{ Por el estilo de la animación } & 162 & $46.2 \%$ \\
\hline \multicolumn{2}{|c|}{ Por las escenas de acción o de peleas } & 156 & $44.5 \%$ \\
\hline \multicolumn{2}{|c|}{ Porque las historias eran "serias" y con riesgos "reales" } & 74 & $21.1 \%$ \\
\hline \multicolumn{2}{|c|}{ Porque no aprecian letras sino caracteres o grafias (ideogramas) } & 17 & $4.8 \%$ \\
\hline \multicolumn{2}{|c|}{$\begin{array}{l}\text { Por influencia de un familiar o amigo/a que me recomendó } \\
\text { verlo }\end{array}$} & 12 & $3.4 \%$ \\
\hline \multicolumn{2}{|c|}{ Por los personajes y lo que me hacían sentir } & 5 & $1.4 \%$ \\
\hline \multicolumn{2}{|c|}{ Por lo que mostraban de la cultura japonesa } & 4 & $1.1 \%$ \\
\hline
\end{tabular}


Por influencia de otro producto cultural relacionado (dorama, manga)

Porque vivía sola y quería ver algo diferente

Porque era lo que transmitía la TV

Fuente: elaboración propia
2

$0.57 \%$

1

$0.28 \%$

1

$0.28 \%$

La Tabla 2, evidencia los primeros acercamientos a este tipo de programación y las razones que llamaron la atención para su consumo, siendo esto último los resultados de una pregunta de respuesta múltiple. El primer dato relevante es la relación inversamente proporcional entre la edad y la iniciación al consumo de animación japonesa. En la muestra, de forma dominante se registran los primeros visionados de anime en la infancia (6-9 años, 44.9\%), seguidos de en la preadolescencia (10-13 años, 29.1\%) y adolescencia (14-17 años, 20\%), mostrando una disminución progresiva a medida que la edad incrementa, siendo la adultez la que registra la menor proporción (1.1\%). Esto puede explicarse por el estereotipo cultural de Occidente acerca de la animación como un producto de consumo infantil, cuestión que, como se mencionó previamente, es errónea, pues hay anime para todo tipo de público independientemente de su edad. De acuerdo con estos resultados, se podría afirmar que si el consumo de anime no surge a temprana edad es inusual que luego se dé en edades más avanzadas.

La exposición al anime a edad temprana se puede relacionar con el concepto de la "niñera televisión", teniendo en cuenta que la tecnología se ha convertido en un apoyo para el entretenimiento de los infantes. En este caso, la televisión cumple el papel de herramienta que ayuda a los padres y madres a criar a sus hijos e hijas manteniéndolos distraídos mientras se realizan otro tipo de actividades como los quehaceres del hogar o trabajar, fomentando así desde la infancia las predisposiciones y preferencias de las personas por determinados programas televisivos. Justamente el aspecto del entretenimiento fue mencionado por casi la mitad de los encuestados (46.8\%) al compararlo con otro tipo de caricaturas.

Sobre las razones por las que los encuestados empezaron a ver anime, más de la mitad (58.8\%) concuerda en que fue porque eran series animadas completamente diferentes a las que sus contrapartes estadounidenses les tenían acostumbrados, considerando que eran diferentes a otro tipo de caricaturas, con historias originales y una manera realista de involucrar temas serios de la vida. El nivel estético también jugó un papel importante pues cerca de la mitad de los encuestados mencionaron que el estilo del dibujo (48\%) y el tipo de animación (46.2\%) contribuyeron en su gusto por este tipo de series.

Tabla 3. Posicionamiento de anime

Top 10 géneros favoritos de anime

\begin{tabular}{|c|c|c|}
\hline Género & Resultado & Porcentaje \\
\hline Shōnen & 290 & $82.9 \%$ \\
\hline Gore & 161 & $46 \%$ \\
\hline Seinen & 147 & $42 \%$ \\
\hline Shōjo & 143 & $40.8 \%$ \\
\hline
\end{tabular}




\begin{tabular}{lcc} 
Mecha & 105 & $30 \%$ \\
Sci-fi & 105 & $30 \%$ \\
Harem & 85 & $24.2 \%$ \\
Ecchi & 83 & $23.7 \%$ \\
Isekai & 79 & $22.5 \%$ \\
Slice of life & 78 & $22.2 \%$ \\
\hline & Top 10 animes favoritos \\
\hline & Resultado & \\
\hline & 168 & Porcentaje \\
Naruto (franquicia)* & 144 & $48 \%$ \\
Dragon Ball (franquicia)** & 80 & $41.1 \%$ \\
One Piece & 67 & $22.9 \%$ \\
Death Note & 62 & $19.1 \%$ \\
Nanatsu no Taizai & 62 & $17.7 \%$ \\
Shingeki no Kyojin & 52 & $17.7 \%$ \\
InuYasha & 49 & $14.8 \%$ \\
Boku no Hero Academia & 46 & $14 \%$ \\
Fullmetal Alchemist & & $13.1 \%$ \\
(franquicia)*** & & \\
Saint Seiya**** & Re.2\% \\
\hline
\end{tabular}

* Incluye Naruto, Naruto Shippuden y Boruto

** Incluye Dragon Ball, Dragon Ball Z, Dragon Ball GT, Dragon Ball Z Kai, Dragon Ball Super.

*** Incluye Fullmetal Alchemist, Fullmetal Alchemist Brotherhood (remake).

$* * * *$ Conocido en Latinoamérica como Los Caballeros del Zodíaco. Incluye Saint Seiya, Saint

Seiya: The Hades Chapter, Saint Seiya Omega, Saint Seiya Lost in Canvas, etc.

Fuente: elaboración propia

La Tabla 3 muestra que el género más consumido por los encuestados es el shōnen, siendo este también el que encabeza la lista del Top 10 de anime favoritos. Este género está dirigido a jóvenes adolescentes masculinos principalmente, el resultado quizás se deba a que la muestra estuvo conformada mayormente por hombres (59.4\%). Sin embargo, cabe aclarar que, aunque demográficamente los shōnen anime sean considerados "para chicos", esto no excluye a las chicas de consumirlos también, un ejemplo de esto puede ser el caso de InuYasha, un anime "de chicos" con una gran base de mujeres fans entre la muestra (3.71\%). Así, respecto a los favoritos, se encontraron anime del género shōnen como: Naruto, Dragon Ball, One Piece, Boku No Hero Academia, Saint Seiya y Nanatsu no Taizai, todos ellos de temática de acción y aventuras o nekketsu; Death Note, un thriller con temática paranormal; InuYasha, que mezcla los géneros de aventuras; Shingeki no Kyojin y Fullmetal Alchemist, de fantasías oscuras con una marcada estética steampunk. 


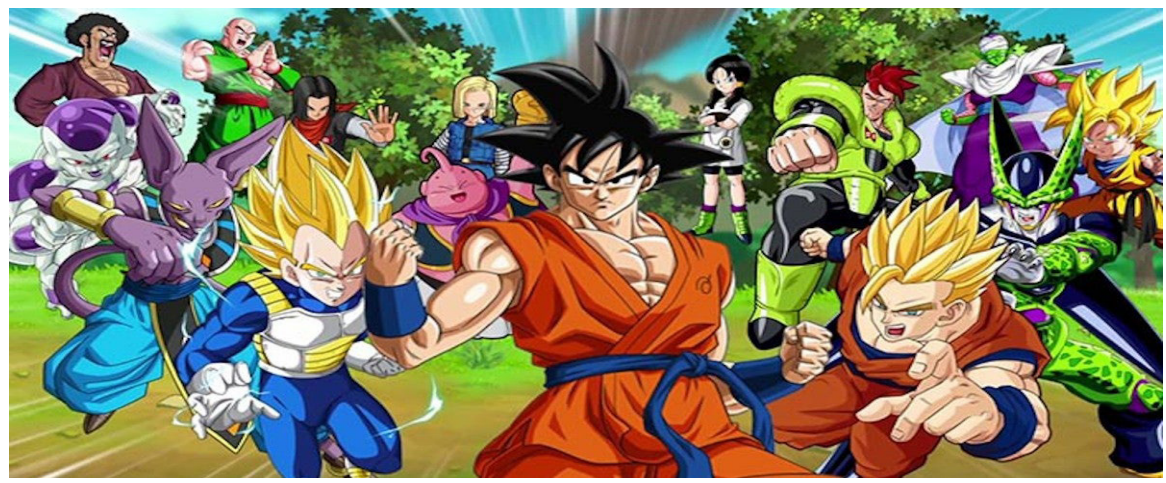

Tomado de: https://lanetaneta.com/necesitas-ver-dragon-ball-z-antes-de-dragon-ball-super/

Sobre estos se pueden destacar varias cosas: Por un lado, los anime (incluyendo sus franquicias) como Dragon Ball, Naruto, Saint Seiya (Los Caballeros del Zodíaco), One Piece, InuYasha y Fullmetal Alchemist, cuentan con una fuerte tradición de consumo en Colombia. Son series animadas con doblaje al español latino, que han sido transmitidas por la televisión colombiana (ya sea abierta o por cable); y que incluso luego de varias décadas en emisión, tal es el caso de Naruto y Dragon Ball, continuaban al aire en el 2020.

En la década del 2000, época que la mayoría de los encuestados registran como sus primeros acercamientos al mundo anime (6-9 años), la televisión colombiana emitía de forma casi simultánea estas series, por lo que eran fáciles de sintonizarlas y seguirlas diariamente. Además, contaron con merchandising dirigido al público masivo (no necesariamente aficionado), especialmente a infantes: desde juegos de cartas, álbumes de calcomanías, tazos en mecatos, hasta todo tipo de material escolar; tal como sucedió, por ejemplo, en 1993 que se hizo el lanzamiento de Los Caballeros del Zodíaco (Saint Seiya) en Televisión Azteca, canal mexicano recibido en Colombia a través de televisión por suscripción, como una estrategia mercantilista para la venta de los productos relacionados a la serie (Lozano, 2007, p. 83).

Por otro lado, existen anime de emisión más reciente que nunca han sido transmitidos por la televisión colombiana y que carecen en muchos casos de doblaje al español latino y sin embargo, gozan también de popularidad. Esto podría explicarse por el cambio en el cómo se consumen los programas de televisión en la última década en la que el auge de la tecnología trajo consigo el nacimiento del streaming, es decir, una nueva forma de transmitir y consumir contenidos audiovisuales mediante plataformas como Netflix, HBO Plus, Amazon Prime Video, Hulu, entre otras, las cuales emiten contenidos de la televisión más diversificados y brindando un mayor dominio de elección a quien se expone, tanto en el contenido como en la manera de consumirlo. La televisión tradicional, de esperar pasivamente los horarios de transmisión de los programas favoritos, pierde incidencia en las vidas de los nuevos consumidores frente a la elección activa de contenido que ofrecen los servicios de streaming. (Suárez-Cousillas et al, 2019).

De esta forma, series como Shingeki no Kyojin y Boku no Hero Academia, que si bien no han sido transmitidas por la televisión tradicional, sí hacen parte de los contenidos ofrecidos por Crunchyroll, una de las grandes plataformas pagas de difusión de contenido anime. De forma similar, pero más abierta al público masivo, están los casos de Nanatsu no Taizai y Death Note que se encuentran dentro del catálogo de series de Netflix, la plataforma de streaming paga con mayor número de consumidores en el 2020 siendo inclusive dobladas también al español latino.

Otra característica que destacar de la Tabla 3 es que de los shōnen mencionados, más de la mitad: Naruto, Saint Seiya, Dragon Ball, Nanatsu no Taizai, Boku no Hero Academia y One Piece, corresponden al género nekketsu: un género del anime-manga que traduce literalmente "sangre 
caliente", nombre que hace referencia a la energía inagotable, espíritu imbatible y temple de acero de los protagonistas, siempre dispuestos a darlo todo por sus ideales. Son series de peleas y aventuras con similitudes y características particulares en cuanto al desarrollo de las historias, personajes y construcción de universo. Así, estos anime transcurren en mundos ficticios, siguen las aventuras de un grupo de amigos en su camino por alcanzar objetivos planteados desde el inicio de la serie y que marcan el plot de la historia, manejan sistemas de combate basados en poderes provenientes de su fortaleza del espíritu: como el chakra, el cosmos, la magia, el ki, los quirks y el haki, y plantean la existencia de elementos particulares y poderosos importantes dentro de la trama como: las frutas del diablo, las esferas del dragón y las bestias con cola (García, 2011).

Cabe destacar que sus historias se basan en el viaje del héroe mostrando el periplo de unos personajes que, si bien desde el comienzo se plantean como especiales (por poseer poderes particulares 0 actitudes no comunes en su universo), inician siendo jóvenes e inexpertos y a medida que avanza su viaje, evolucionan y maduran, tanto como luchadores, como personas. Más importante aún, en estos anime es que profesan valores de importancia para la sociedad japonesa (y en general) representando mensajes como el nunca rendirse, luchar por los sueños, sobreponerse a la derrota y mejorar a través del estudio y la práctica, así como el siempre apoyar y luchar por los amigos (Sayar, 2017, p.177).

Tabla 4. frecuencia de consumo diario

\begin{tabular}{lcccc}
\hline \multicolumn{5}{c}{ Tiempo en días y horas dedicado a ver anime } \\
\hline \multicolumn{1}{c}{ Días } & $\begin{array}{c}\text { De } 0 \text { a } 3 \\
\text { horas }\end{array}$ & $\begin{array}{c}\text { De } 4 \text { a } 7 \\
\text { horas }\end{array}$ & $\begin{array}{c}\text { De } 8 \text { horas en } \\
\text { adelante }\end{array}$ & Total \\
Lunes & $\begin{array}{c}323 \\
(92.2 \%)\end{array}$ & $24(6.9 \%)$ & $3(0.9 \%)$ & \\
Martes & 316 & $31(8.9 \%)$ & $3(0.9 \%)$ & \\
Miércoles & $308(88 \%)$ & $39(11.1 \%)$ & $3(0.9 \%)$ & \\
Jueves & 311 & $37(10.6 \%)$ & $2(0.6 \%)$ & \\
Viernes & $(88.8 \%)$ & & & \\
Sábados & $11(3.1 \%)$ & $261(74.6 \%)$ & $78(22.3 \%)$ & $350(100 \%)$ \\
& 190 & $127(36.3 \%)$ & $33(9.4 \%)$ & \\
Domingos & $(54.3 \%)$ & & & \\
& 200 & $117(33.4 \%)$ & $33(9.5 \%)$ & \\
\hline
\end{tabular}




\begin{tabular}{lcc}
\hline \multicolumn{3}{c}{ Momento del día en el que ven anime } \\
\cline { 1 - 1 } \multicolumn{1}{c}{ Momento del } & Resultado & Porcentaje \\
Noche & 247 & $70.6 \%$ \\
Tarde & 70 & $20 \%$ \\
Madrugada & 23 & $6.6 \%$ \\
Mañana & 10 & $2.8 \%$ \\
\hline Total & 350 & $100 \%$ \\
\hline
\end{tabular}

Fuente: elaboración propia

La Tabla 4, frecuencia del consumo, describe los momentos del día predilectos para ver anime así como la intensidad horaria y diaria de los mismos. Se registra un consumo estable de 0 a 3 horas de lunes a jueves, entre $88 \%$ a $92.2 \%$, y un aumento significativo y constante, de 4 a 8 horas y en adelante durante los fines de semana (sábados y domingos), siendo el viernes el día donde se produce un comportamiento atípico en el consumo por parte de la mayoría de los encuestados $(74.6 \%)$, focalizado en una exposición de 4 a 7 horas. Sobre el momento del día preferido para ver anime la noche destaca con un $70.6 \%$ de los resultados.

El contraste entre pocas y muchas horas dedicadas al anime entre lunes a jueves, viernes y sábados a domingo respectivamente podría ser explicado como una consecuencia del mismo estilo de vida de la población universitaria. Pues estos, ya sean estudiantes o docentes, de lunes a jueves se encuentran en virtud de las labores y responsabilidades propias de las clases. Cuestión que cambia de viernes a domingos que, entrados al fin de semana, la rutina pasa a enfocarse más en los planes sociales y/o referentes a hobbies. A su vez, esto explicaría también la preferencia por la noche como momento del día de visionado de anime pues, normalmente y salvo excepciones, la vida productiva universitaria ocurre de día mientras que la noche se reserva para descansar o dedicarlo a actividades de esparcimiento.

Sobre la frecuencia de visionado entra a relucir un concepto importante, los heavy viewers, que de acuerdo a lo señalado por la teoría del Cultivo, hace referencia a aquellos individuos que tienen mayor frecuencia de exposición a ciertos contenidos televisivos. En este caso vendrían siendo los participantes que indicaron dedicarle al consumo de anime de 8 horas en adelante. Teniendo en cuenta la teoría, la constante exposición a este tipo de contenidos los hace más susceptibles a producir en ellos efectos significativos que fomentan la base para el cultivo de ciertas creencias, perspectivas y valores. De esta forma se puede inferir, según los resultados expuestos por la muestra, que aquellos individuos con mayores posibilidades de ser influenciados por los mensajes transmitidos por la animación japonesa son aquellos que registran altas horas de visionado los fines de semana. Los heavy viewers que se concentran viernes, sábado y domingo. 
Tabla 5. Percepción de impacto de consumo

\begin{tabular}{|c|c|c|}
\hline \multicolumn{3}{|c|}{ Ver anime te ha motivado a } \\
\hline Respuesta & Resultado & Porcentaje \\
\hline A meditar sobre mi vida, hacer introspección & 198 & $56.6 \%$ \\
\hline Dibujar al estilo manga, hacer fanarts, o dōjinshi* & 130 & $37.1 \%$ \\
\hline A estudiar sobre cultura y costumbres japonesas & 112 & $32 \%$ \\
\hline Ir a convenciones sobre el tema & 106 & $30.2 \%$ \\
\hline Ver programas de televisión japoneses (Ej: dorama) & 102 & $29.1 \%$ \\
\hline Ir a restaurantes de comida japonesa & 96 & $27.4 \%$ \\
\hline Ver cine japonés & 91 & $26 \%$ \\
\hline Practicar un deporte & 85 & $24.2 \%$ \\
\hline Viajar a Japón a hacer turismo & 81 & $23.1 \%$ \\
\hline Aprender a cocinar platos japoneses & 68 & $19.4 \%$ \\
\hline Tomar un curso de idioma japonés & 67 & $19.1 \%$ \\
\hline Aprender a tocar un instrumento musical & 48 & $13.7 \%$ \\
\hline Aprender manualidades (Ej: origami) & 48 & $13.7 \%$ \\
\hline Hacer animación & 45 & $12.8 \%$ \\
\hline Viajar a Japón a estudiar & 39 & $11.1 \%$ \\
\hline
\end{tabular}

* dōjinshi se refiere a mangas autopublicados. Generalmente las historias consisten en una parodia o historia original, pero con personajes de animes o mangas que ya existentes. Son creados por personas que quieren publicar sus propios cómics.

Fuente: elaboración propia

La Tabla 5, evidencia la influencia de la animación japonesa percibida por los aficionados cartageneros encuestados. Esta representa los resultados de una pregunta de opción múltiple por lo que un mismo participante podía identificar más de una influencia, además de cruzar estos resultados con una serie de testimonios descritos por los mismos.

El primer dato por rescatar es que parte de las principales influencias de la animación japonesa es la de despertar la curiosidad sobre otros elementos de la cultura nipona. Los encuestados muestran haberse visto atraídos por el estilo de vida, gastronomía y artes propios del país, inspirándose a consumir su comida tradicional $(27.4 \%)$, sus otros tipos de programas de televisión y su cine $(55.1 \%)$. También, desde una postura más proactiva, poco más de la mitad $(52.2 \%)$ mostraron verse motivados a estudiar la cultura de Japón: su idioma, el cocinar sus platillos tradicionales y realizar sus manualidades (origami). Incluso dibujar al estilo manga, hacer fanarts y doujinshis (37.1\%) e inspirarse a viajar al país como turista o estudiante (34.2\%): “(..) me ha inspirado a leer, a investigar acerca de la cultura japonesa y sus costumbres, me ha motivado a desarrollar habilidades artísticas mediante el dibujo, y ha despertado mi imaginación (...)" (estudiante hombre, 20-25 años).

Cabe resaltar que más de la mitad (56.6\%) de los encuestados manifestaron que el ver anime los ha motivado a hacer introspección y a meditar sobre sus vidas. Esto se explica mediante la Teoría del Cultivo, pues esta indica que entre más expuestas están las personas a ciertos contenidos, en 
este caso la animación japonesa, mayor predisposición tendrán a desarrollar perspectivas, valores y estilos de vida similares a las representaciones a las que se ven expuestos: "El anime fue justo lo que necesitaba cuando niña para reforzar un sistema de valores que me permitió avanzar de una forma diferente a la ordinaria. La responsabilidad, la perseverancia y el espíritu luchador, valores característicos de los japoneses, se encuentran embebidos en cada capítulo y cada personaje, de manera que te llegan al corazón y te motivan a ser mejor sin que siquiera te des cuenta" (estudiante mujer, 17-19 años). Este testimonio evidencia una filosofía muy arquetípica en anime del género nekketsu predominante en el top 10 de anime favoritos.

Los resultados permiten validar lo planteado por la Teoría del Cultivo cuando señala que si las imágenes y valores presentes en los contenidos visionados se repiten con un alto grado de constancia, influyen en la cosmovisión de quienes se exponen, cultivando y/o reforzando concepciones semejantes entre estos productos audiovisuales y la realidad. Los participantes de la encuesta muestran asociar con su vida la cultura nipona a través de sus contenidos animados, a la vez que implementan o adoptan aquello que creen les sirve para su diario vivir.

\section{Conclusiones}

La noción de "cultivo" hace referencia a aquello que de manera independiente contribuye a la concepción que se forman las personas sobre su entorno social como un efecto a la exposición regular a determinados contenidos. De esta manera, la teoría del Cultivo halló que sí existe influencia de estos a los que los individuos se exponen reflejado en las actitudes, creencias y perspectivas, lo que se alinea con los resultados de este estudio.

En el análisis se encontró una variable importante frente al impacto que ha tenido el anime en los encuestados, el tiempo de visionado, en donde se destacan los heavy viewers, o espectadores pesados, aquellos quienes manifestaron tener un alto grado de exposición dedicándole de ocho horas en adelante, así como también el tiempo que llevan consumiéndolo, como mínimo once años y en algunos casos más de veinte (la diferencia entre la edad en la que manifestaron iniciar el consumo y la edad que tenían al responder el cuestionario). Lo anterior, explicaría la susceptibilidad de los encuestados a los efectos significativos que fomentaron la base para el cultivo de creencias, perspectivas y valores, ya que, teniendo en cuenta la teoría del Cultivo, una exposición constante a este tipo de contenidos genera mayor susceptibilidad a ser influenciados por aquello que consumen.

Se encontró evidencia de que gran parte de la muestra inició el visionado de anime a una edad temprana (6-9 años) como forma de entretenimiento. Esto puede explicarse desde el concepto de la "niñera televisión", es decir, el uso que los padres dan a este aparato como forma de entretener a los hijos pequeños, considerando estos a su vez a la animación japonesa como un producto apto para consumo infantil. Sin embargo es de considerar que hay animes dirigidos a adolescentes, jóvenes y adultos, pero al parecer en Colombia todos están catalogados como caricaturas aptas para infantes, aspecto que merece una revisión por parte de las programadoras.

Se encuentra que la exposición de los encuestados al anime pasó de considerarse entretenimiento a una forma de aprendizaje y crecimiento personal. Estos manifiestan haber desarrollando valores, creencias y estilos de vida similares a los representados en los animes. Se les despertó el interés por elementos propios de la cultura nipona, traducido en querer degustar y preparar su gastronomía, ver su cine y otros tipos de shows televisivos, hasta el deseo por aprender su idioma, viajar a este país y aprender a dibujar manga. 
Se espera que este estudio permita avanzar en el análisis del tema y su categorización para lo que se sugieren entrevistas a profundidad con este tipo de consumidores.

\section{Referencias}

Agencia de Noticias Univalle (2018, 25 de septiembre). El impacto de la cultura visual japonesa. Recuperado de http://emisora.univalle.edu.co/el-impacto-de-la-cultura-visual-japonesa/

Arciniegas, S. y Mora, S. (2010). Estudio del diseño del anime japonés como generador de prácticas placenteras (tesis de pregrado). Universidad Sergio Arboleda. Bogotá, Colombia. Recuperado de https:// en.calameo.com/read/002357685a554cd00c8b1

Banrepcultural - Red Cultural del Banco de la República de Colombia (s.f). La televisión en Colombia. Recuperado de https://enciclopedia.banrepcultural.org/index.php/La televisi\%C3\%B3n_en_Colombia\#Los nuevos_canales_privados_1998

Cobos, T. (2010). Animación japonesa y globalización: la latinización y la subcultura otaku en américa latina. Razón y Palabra, 72. Recuperado de http://www.razonypalabra.org.mx/N/N72/Varia_72/32_Cobos_72.pdf

Conejo, F. (2011). Japón hecho en Venezuela: influencia cultural japonesa en la cosmovisión del mundo de los jóvenes en Venezuela a través de la televisión y el anime. XIII Congreso Internacional de la Asociación Latinoamericana de Estudios de Asia y África - ALADAA. Bogotá, Colombia. Recuperado de https://ceaa. colmex.mx/aladaa/memoria_xiii_congreso internacional/images/conejo.pdf

D’Adamo, O., García, V. y Freidenberg, F. (2007). Medios de comunicación y opinión pública. Madrid: Editorial McGraw Hill/Interamericana de España, S.A.U.

Flores Guevara, S., \& Hernández Téllez, J. (2019). Realidad Virtual \& Género. Vocaloid versus Hatsune Miku, una "diva androide". LUCIÉRNAGA, 10(19). Recuperado de: https://revistas.elpoli.edu.co/index.php/ luc/article/view/1466/1109

El Tiempo (2001, 16 de diciembre). Muñecos malditos. Recuperado de https://www.eltiempo.com/archivo/ documento/MAM-715005

Galindo, Y. (2017). Influencia de los animes japoneses en los jóvenes de la asociación cultural A-Shinden Puno 2016 (tesis de pregrado). Universidad Nacional del Altiplano, Puno, Perú. Recuperado de _ http://repositorio. unap.edu.pe/bitstream/handle/UNAP/3518/Galindo_Velasquez_Yanett.pdf?sequence=1\&isAllowed=y

García, J. (2011). La épica y las sagas fantásticas en el manga: un acercamiento a la traducción argentina. Digilenguas, 10, 66-76. Recuperado de https://editorial.fl.unc.edu.ar/book-review/digilenguas-10/

Hernández, R., Fernández, C. y Baptista, P. (2014). Metodología de la investigación. (6ta. Ed.). México: Editorial McGraw Hill Education.

Igartua, J. y Humanes, M. (2004). Teoría e investigación en comunicación social. Madrid: Ediciones Síntesis, S.A.

IGN Latinoamérica (2019, 18 de diciembre). Anime: la industria rompe récord en ganancias por sexto año consecutivo. Recuperado de: https://latam.ign.com/anime/65881/news/anime-la-industria-rompe-record-enganancias-por-sexto-ano-consecutivo

Intriago, D. (2018). Análisis de la influencia cultural del anime japonés en la recepción comunicológica de estudiantes de primer y tercer semestre de Diseño Gráfico de la Universidad de Guayaquil (tesis de pregrado). Universidad de Guayaquil, Guayaquil, Ecuador. Recuperado de http://repositorio.ug.edu.ec/ bitstream/redug/20941/1/TT\%20Intriago\%20C\%C3\%B3rdova\%20Dialmar\%201.pdf 
Lozano, J. (2007). Teoría e investigación de la comunicación de masas. México, D.F: Pearson Educación.

Shock (2019, 25 de marzo). Los mejores episodios de Cuentos de los Hermanos Grimm para pasar el guayabo. Recuperado de https://www.shock.co/cine-y-tv/los-mejores-episodios-de-cuentos-de-los-hermanos-grimmpara-pasar-el-guayabo-ie2636

Pichón, M. (2012). El anime y la apropiación de la cultura japonesa por parte del otaku barranquillero (tesis de pregrado). Universidad del Norte, Barranquilla, Colombia. Recuperado de https://www.academia. edu/30611346/EL_ANIME Y LA APROPIACION_DE_LA CULTURA_JAPONESA_POR_PARTE_DEL_ OTAKU BARRANQUILLERO

Romero, J. (2012). Influencia cultural del anime y manga japonés en México (tesis de pregrado). Universidad Autónoma del Estado de México, Toluca, México. Recuperado de http://ri.uaemex.mx/bitstream/ handle/20.500.11799/214/Influencia_cultural_del_anime.pdf?sequence=1

Sayar, R. (2017). Testigos antiguos en épocas modernas: el martirio en Saint Seiya a la luz de IV Macabeos. Congreso Nacional de la Asociación Latinoamericana de Estudios de Asia y África - ALADAA 2017. Buenos Aires, Argentina. Recuperado de http://aladaa.com.ar/asiayafrica/wp-content/uploads/2018/12/2018.ACTAS.-DOCUMENTO-7.pdf

Suárez-Cousillas, T., Martínez-Fernández, V. y Sánchez-Amboage, E. (2019). Audiencias de plataformas SVOD: el caso de Netflix, Blockbuster, Hulu y HBO. 14th Iberian Conference on Information Systems and Technologies. Coimbra, Portugal. Recuperado de https://www.researchgate.net/publication/334604413 Audiencias de plataformas SVOD_El_caso_de Netflix_Blockbuster_HUlu_y_HBO

Zarco, L. (2017). Apropiación de dramas coreanos en un grupo de jóvenes de Cartagena de Indias (tesis de pregrado). Universidad de Cartagena, Cartagena de Indias, Colombia. Recuperado de http:// repositorio.unicartagena.edu.co/bitstream/handle/11227/6157/TRABAJO\%20DE\%20GRADO\%20LAZQ. pdf?sequence $=1$

\section{Notas}

[1] Cosplay viene de costume-play, consiste en caracterizar personajes de ficción a través de disfraces, trajes y accesorios, así como el personificar mediante el acting la personalidad del mismo.

\section{Para citar este artículo}

Rodríguez, N., Arias, F.; Cobos, T. (2020). Exposición y efectos del consumo de anime en la población universitaria de Cartagena. Revista Luciérnaga Comunicación. Vol.12 Núm. 23. Pp 69-87. https://doi.org/10.33571/revistaluciernaga.v12n23a3

OJS. http://revistas.elpoli.edu.co/index.php/luc/issue/archive

Link. https://www.politecnicojic.edu.co/index.php/revista-luciernaga 